\title{
Pulmonary alveolar proteinosis and successful therapy with combined lavage procedures: Case reports
}

\author{
NALAN DEMIR FIRAT ${ }^{1}$, AYDIN ÇILEDAG $\breve{G}^{1}$, PINAR AKIN KABALAK ${ }^{1}$, DEMET KARNAK $^{1}$, \\ BAŞAK CEYDA MECO ${ }^{2}$, ZEKERIYYA ALANOĞLU ${ }^{2}$ and NESLIHAN ALKIŞ ${ }^{2}$
}

Departments of ${ }^{1}$ Chest Diseases, ${ }^{2}$ Anesthesiology, Ankara University School of Medicine, Ankara, Turkey

Received February 2, 2011; Accepted March 16, 2011

DOI: $10.3892 / \mathrm{etm} .2011 .230$

\begin{abstract}
Pulmonary alveolar proteinosis (PAP) is a rare disease characterized by the accumulation of lipoproteinaceous material within alveolar spaces. Whole-lung lavage (WLL) has been the most common therapeutic intervention for this disorder. However, patients presenting with PAP are usually hypoxemic or in poor clinical condition, and WLL may be impossible to perform. In such cases, multiple segmental lavage (MSL) may be advocated as a first-choice therapy prior to WLL. Herein, we present two cases with idiopathic PAP treated successfully with both lavage techniques consecutively. After the MSL procedure, WLL was performed, and both patients showed a marked clinical and physiologic improvement. Therefore, for patients who are not good candidates for general anesthesia, we recommend MSL (or 'prewash') before WLL to produce an increase in the blood oxygen level for long-duration general anesthesia. In the surgical room, close monitoring and repositioning of the patient as well as maintenance and inspection of the correct tube position, and manual chest wall percussion are extremely important for the safety and success of the procedure.
\end{abstract}

\section{Introduction}

Pulmonary alveolar proteinosis (PAP) is a rare disorder first described in 1958 by Rosen et al (1). Alveolar spaces are progressively filled with a phospholipoproteinaceous material presumably caused by malfunction of the balance between surfactant production by type II pneumocytes and surfactant removal. The latter is affected primarily by alveolar macrophages. A diagnosis of PAP can be confirmed by typical histopathological findings of lung biopsy specimens or the appearance of bronchoalveolar lavage. Whole-lung lavage (WLL) introduced by Ramirez in the late 1960s, is still the gold

Correspondence to: Professor Demet Karnak, Department of Chest Diseases, Ankara University School of Medicine, 06100 Cebeci, Ankara, Turkey

E-mail: demet.karnak@gmail.com

Key words: pulmonary alveolar proteinosis, whole-lung lavage, multiple segmental lavage standard therapy (2). Therapy with granulocyte macrophage colony-stimulating factor (GM-CSF) is another option, but its long-term safety has not yet been confirmed (3). The severity and natural history of alveolar proteinosis is variable, and severe hypoxemia may occur during the course of the disease. In patients with poor clinical condition and hypoxemia, WLL is difficult to perform due to possible complications involving general anesthesia. WLL often requires more than 4 hours per lung to perform (4). For surgery, the time is longer, and complication rates can be high. In these patients, multiple segmental lavage (MSL) with flexible bronchoscopy (FB) can be initially carried out to prepare the patient for the long-lasting general anesthesia required for WLL.

\section{Materials and methods}

Multiple segmental lavage. In our technique, FB is wedged as accurately as possible in all of the segments without error during the procedure. Before and during MSL, 2\% xylocaine and low-dose parenteral sedation with midazolam and phentanyl are administered. In general, the lavage is preferably carried out on the lung part or lobe noted to have the most extensive accumulation as determined by radiology. While the patient breaths oxygen through a nasal cannula, a FB is passed through the mouth and placed in each segmental bronchus. Warm saline solution is instilled via a syringe in $50-\mathrm{ml}$ aliquots and is removed by suction. The returning lavage fluid is initially milky and gradually becomes clear. The tip of the FB is then switched to another segment of the orifice. Segments or subsegments of all of the lobes in the right or left lung are cleaned one by one using this method. As it is successful and minimally stressful for the physician and patient, MSL can be referred to as 'prewash'.

Whole-lung lavage. In our technique, WLL of the preferred lung is performed under general anesthesia via isolation of the two lungs with a double-lumen endotracheal tube. Performance of single-lung ventilation lasts for a duration of 5 to 10 hours. The correct positioning of the tube is confirmed by fluoroscopy and fiberoptic bronchoscopy. Invasive arterial access for frequent blood gas analysis and continuous blood pressure monitoring should be carried out in addition to routine anesthesia monitoring which consists of $\mathrm{O}_{2}$ saturation, $\mathrm{CO}_{2}$ level and gas analysis and ECG. Two large bore (18-G) 
IV access sites are initiated immediately after induction of anesthesia for rapid infusion of fluids. Central venous access is not preferred due to the risk of pneumo-hemothorax during the insertion of the catheter. Anesthesia induction can be performed with propofol $2 \mathrm{mg} / \mathrm{kg}$, remiphentanyl $1 \mu \mathrm{g} / \mathrm{kg}$, and rocuronium $0.6 \mathrm{mg} / \mathrm{kg}$, and management is recommended to be continued with sevoflurane $2 \%$ in $50 \%$ oxygen $/ 50 \%$ air, remi phentanyl $0.01 \mu \mathrm{g} / \mathrm{kg} \mathrm{IV}$ bolus, when needed. Hemodynamic and respiratory parameters of the patient should be monitored throughout the procedure. Both lungs should be ventilated with $100 \%$ oxygen for $10 \mathrm{~min}$ for the denitrogenation process. The isolation of each lung is confirmed by water seal testing. Since the residual nitrogen bubbles can diminish the access of lavage fluid to the alveolar space and consequently the efficacy of WLL, after separation of the lungs, the lumen of the endotracheal tube leading to the lung to be lavaged is clamped proximally at the end of expiration (at functional residual capacity) for $5 \mathrm{~min}$ to achieve adequate degassing. Warmed isotonic saline solution at $37^{\circ} \mathrm{C}$ is then instilled by gravity $50 \mathrm{~cm}$ above the carina. After filling the lung with approximately $800 \mathrm{ml}$ of saline, the lavage process consists of slowly instilling 500-ml aliquots in each cycle via a catheter. Manual chest percussion should be performed throughout the procedure as it has been noted that chest wall percussion enhances the removal of proteinaceous material. During the procedure, the position of the patient can be changed from supine to left or right lateral position intermittently. The average procedure lasts approximately 5-10 hours. An average range of 12 to 20 liters of warmed saline should be used, and lavage should be discontinued when the returning fluid becomes clear. The patient can be repositioned several times to a lateral decubitis position in relation to the non-lavaged lung. The major concerns which should be considered during repositioning of the patient include the possible malpositioning of the doublelumen tube and ischemic complications of the extremities. Extra precautions should be taken to prevent these complications by checking the positioning of the tube, monitoring the airway pressure changes, air leakage and placing supporting pillows under the thighs, head and axilla.

\section{Case reports}

Case 1. A female patient, 31 years of age, was admitted to the hospital with exertional dyspnea experienced for two years. She had a three pack-year history of smoking. Her dyspnea had progressively increased, and hemoptysis and cough had developed one month earlier. She had never been exposed to occupational dust, and her past medical history was unremarkable. On physical examination, her pulse was $122 / \mathrm{min}$ and respiratory rate, $28 / \mathrm{min}$. Bilateral crackles were audible at both lung bases. On laboratory analyses, the white cell count was $9100 / \mathrm{mm}^{3}$, the erythrocyte sedimentation rate (ESR) $8 \mathrm{~mm} / \mathrm{h}$ and lactate dehydrogenase was $523 \mathrm{mg} / \mathrm{dl}$ (normal range $(\mathrm{N}), 240-480)$. Cholesterol and triglyceride levels were $219 \mathrm{mg} / \mathrm{dl}(\mathrm{N},<200 \mathrm{mg} / \mathrm{dl})$ and $164 \mathrm{mg} / \mathrm{dl}(\mathrm{N},<150 \mathrm{mg} / \mathrm{dl})$, respectively. Blood gas analysis showed moderate hypoxemia $\left(\mathrm{PaO}_{2}, 49.2 \mathrm{mmHg}\right)$. Pulmonary function tests revealed a restrictive pattern with a reduced DLCO (55\%) value. A chest X-ray detected bilateral opacities at the mid-lower zones (Fig. 1). A thoracic high resolution computerized tomography

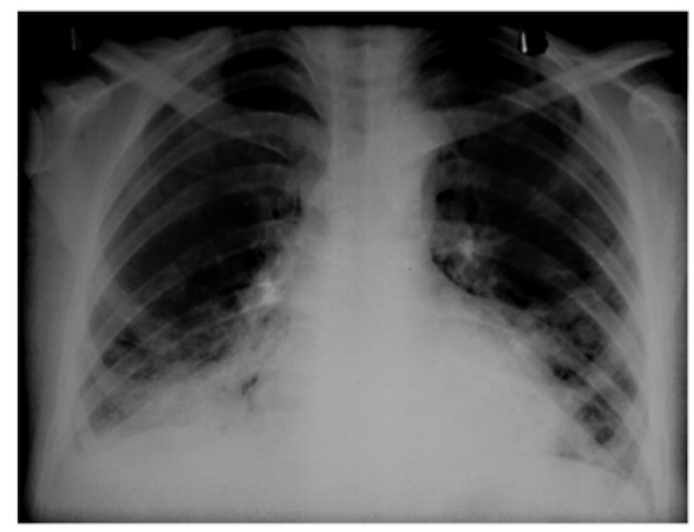

Figure 1. Chest X-ray on admission revealing bilateral infiltration at the middle and lower zones (case 1).

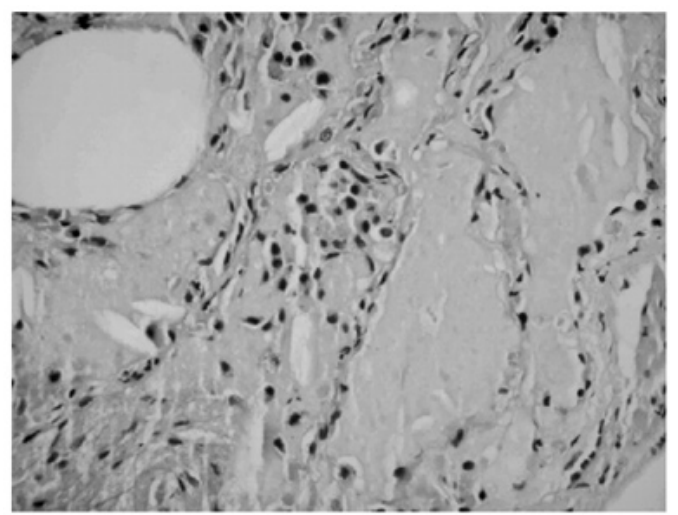

Figure 2. Hematoxylin and eosin staining (x400) of a transbronchial biopsy specimen revealing permeation of the alveolar spaces with amorphous material (case 1).

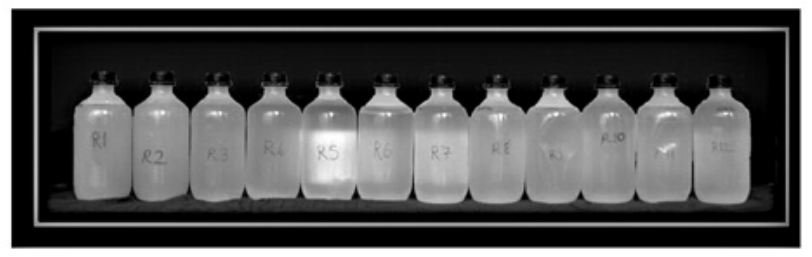

Figure 3. Drained fluids of one lung obtained by WLL in case 1. After the fifth bottle, with chest percussion, the returning fluid became cloudy.

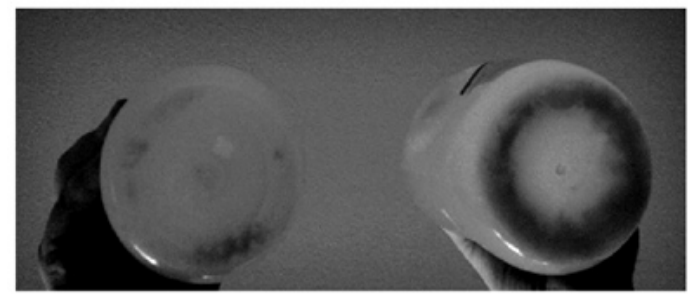

Figure 4. Proteinous materials accumulated at the bottom of the bottle before and after chest wall percussion (case 1).

(HRCT) revealed bilateral diffuse ground glass opacities and interlobular septal thickening. Bronchoalveolar lavage (BAL) fluid revealed a milky turbid appearance. A transbronchial 
lung biopsy (TBLB) was taken, and histopathologic examination revealed PAS (+) lipoproteinaceous material deposition (Fig. 2). Accordingly, a diagnosis of PAP was made. WLL was planned; however, due to the poor clinical condition of the patient with hypoxemia and her relatively high-risk profile, she was not considered as a suitable candidate for general anesthesia. Therefore, MSL under local anesthesia was performed. A total of $2000 \mathrm{ml}$ saline was instilled, and approximately $1600 \mathrm{ml}$ was aspirated from the right lung. MSL lasted approximately 1 hour without any complication. Following MSL, the $\mathrm{PaO}_{2}$ level increased to $60.3 \mathrm{mmHg}$ on breathing ambient air and remained stable until the WLL procedure scheduled a few days later. The patient was re-evaluated and consequently scheduled for general anesthesia.

In total, 13 liters of warmed saline solution were instilled, and 12 liters were obtained (Figs. 3 and 4). Immediately after the procedure, the double-lumen tube was replaced with a single-lumen tube by an anesthesiologist. The patient was transferred to the ICU, and mechanical ventilation was continued for 12 hours. There was no complication except mild hypokalemia and metabolic acidosis.

After one month, WLL of the right lung was performed using the same technique mentioned above. During WLL, severe hypoxia $\left(\mathrm{SpO}_{2}<70 \%\right)$ and an increase in airway pressure $\left(40 \mathrm{cmH}_{2} \mathrm{O}\right)$ developed 2 hours after the initiation of the single-lung ventilation due to malpositioning of the double-lumen tube. The patient was immediately extubated and reintubated with the double-lumen tube, and correct positioning of the tube was confirmed by fluoroscopy and fiberoptic bronchoscopy. The rest of the procedure was uneventful. In this second procedure, a total of 12 liters of saline was instilled, and 11 liters was drained without any complications. After these combined lavage procedures, marked clinical, physiological and radiological improvements (Fig. 5) were observed. $\mathrm{PaO}_{2}$ and diffusion capacity increased to $85 \mathrm{mmHg}$ and $75 \%$, respectively (Fig. 6).

Case 2. A female, 44 years of age, was admitted to the hospital with exertional dyspnea for a one-year period. She had a five pack-year history of smoking and had worked in a cloth factory for five years. There was no significant exposure to organic or inorganic material. On physical examination, her pulse and respiratory rate were $76 / \mathrm{min}$ and $26 / \mathrm{min}$, respectively. Bilateral crackles were audible at both lung bases. On laboratory analyses, the white cell count and ESR were normal. The blood gas analysis showed hypoxemia $\left(\mathrm{PaO}_{2}, 56 \mathrm{mmHg}\right)$, and pulmonary function tests revealed a restrictive pattern with a decreased value of DLCO (58\%). Chest radiograph revealed bilateral infiltrations at the mid-lower zones (Fig. 7) and thoracic HRCT showed bilateral diffuse ground glass opacities and interlobular septal thickening showing a crazy paving pattern. Bronchoalveolar lavage fluid revealed a milky turbid appearance. The diagnosis of PAP was established by open lung biopsy.

A combined lavage procedure was planned as in case 1. MSL was performed using a total of $2600 \mathrm{ml}$ warmed saline for the right lung and $2300 \mathrm{ml}$ for the left lung until blood gases became high enough (above $60 \mathrm{mmHg}$ ) for general anesthesia. Two WLL procedures were performed separated by an interval of 1 month. The first WLL was carried out using

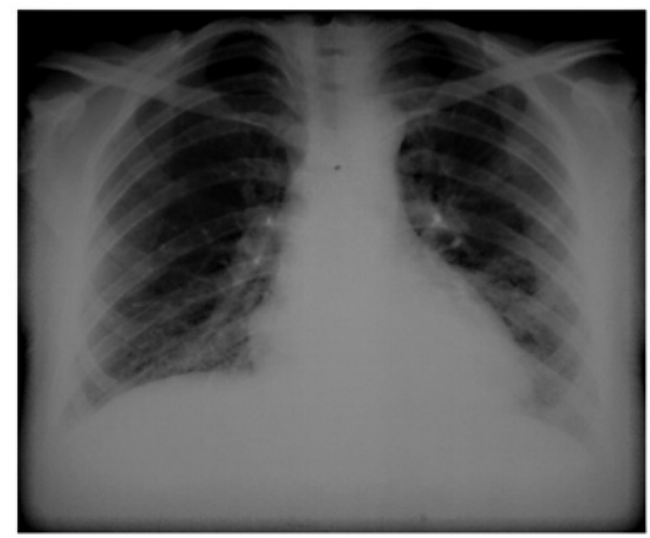

Figure 5. Radiological resolution after therapy in case 1.

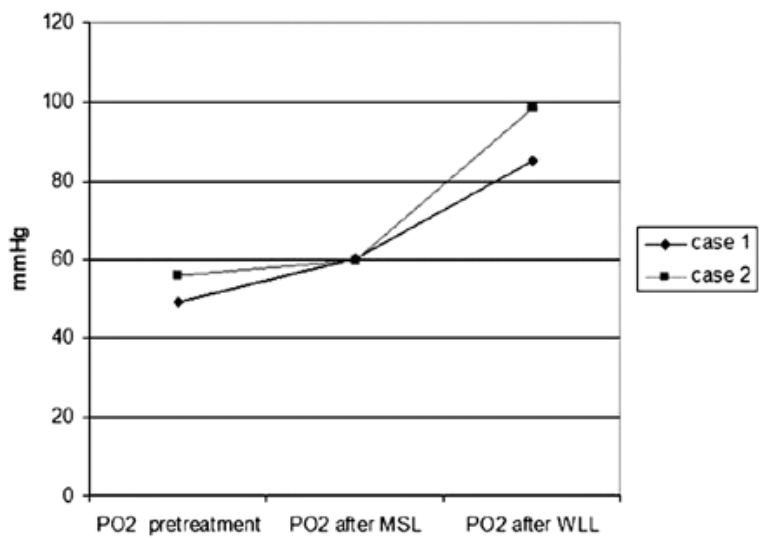

Figure 6. Oxygen pressure readings of cases 1 and 2 indicating the success of the combined lavage procedures.

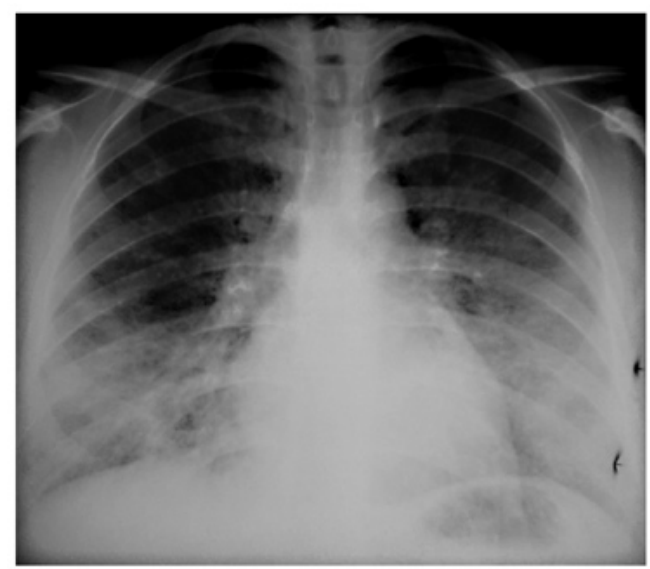

Figure 7. Chest X-ray on admission revealing bilateral infiltration at the middle and lower zones (case 2).

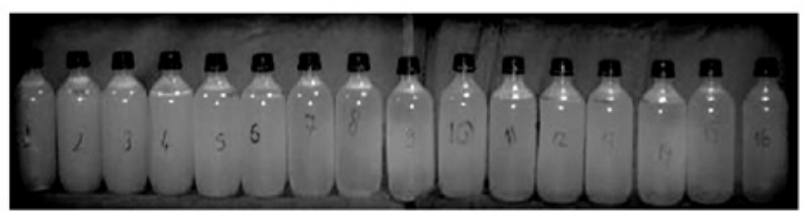

Figure 8. Drained fluids of one lung obtained by WLL in case 2. 


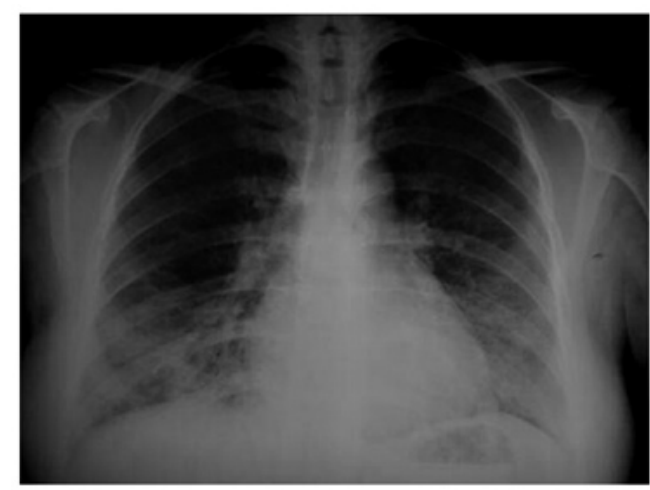

Figure 9. Radiological resolution after therapy in case 2.

17 liters of saline for the right lung (Fig. 8) and the second using 15 liters for the left lung. No complications were noted for either MSL or the WLLs. After the procedures, clinical and radiological improvements (Fig. 9) were noted, and the $\mathrm{PaO}_{2}$ level rose to $98.5 \mathrm{mmH}$ along with increased diffusion capacity (82\%) (Fig. 6).

\section{Discussion}

Pulmonary alveolar proteinosis is a rare disorder characterized by the accumulation of lipoproteinaceous material within the alveolar compartment (1). PAP is classified as congenital, secondary and idiopathic. The pathophysiology of PAP is characterized by several mechanisms; surfactant protein $\mathrm{B}$ mutations and granulocyte monocyte colony-stimulating factor receptor defects, inability of the macrophage to catabolize surfactant, and the presence of certain systemic disorders and exposure to various materials. Idiopathic PAP is rare, with a prevalence of $0.37 / 100,000$ individuals, yet it constitutes $90 \%$ of all cases $(5,6)$. Surgical lung biopsy is the gold standard for the diagnosis of PAP, but in an appropriate clinical setting, a diagnosis can be confirmed by BAL and/or TBB (7). In this report, the diagnosis of PAP was made by TBLB in case 1 and surgical lung biopsy in case 2 . According to the aforementioned descriptions, these two cases were determined to be idiopathic PAP lacking a secondary reason.

According to literature studies, treatment of PAP includes corticosteroids (8), potassium iodide (9), streptokinase (10) and aerosolized trypsin. However, none of these agents has adequate efficacy (11). More recently, therapy with GM-CSF has been attempted. Although a positive effect of GM-CSF has been shown in PAP, its long-term safety has not been determined, and the optimal dose, optimal duration of treatment and the optimal route of GM-CSF remain unclear (12-15).

Whole-lung lavage is now considered to be the most effective treatment for PAP (4,15-16). There are no standard indications for WLL, although the following criteria have been proposed: daily life activity-impairing dyspnea, $\mathrm{PaO}_{2}<60 \mathrm{mmHg}$ and shunt fraction $>10-12 \%$ (6). The major adverse effect of WLL is hypoxemia that can be improved with a high inspired oxygen concentration. Hemodynamic changes can also occur with single-lung ventilation which may necessitate invasive monitoring during the procedure. The major risks of WLL concern the correct placement of the double- lumen endotracheal tube. In the case of wrong replacement, spilling of fluid from the lavaged lung to the ventilated lung can occur. Other complications include pleural collections, hydropneumothorax, barotrauma and hypothermia. Due to these potential complications and since the patients are usually hypoxemic and in poor clinical condition, WLL is frequently impossible to be performed. In such cases, multiple segmental or lobar lavage by FB has been reported as a possible alternative to WLL. In several case reports, it has been reported that MSL is a simple and safe procedure which has led to an improvement in PAP $(3,17)$. However, the fluid yielded by this method is small, and the volume of lavage fluid is limited. Hence, it is useful for patients with less advanced disease (3). In contrast, our cases had advanced disease and poor clinical condition with hypoxemia, thus they were not good candidates for therapy with MSL alone. MSL was carried out initially in order to prepare the patients for WLL. Although there have been many publications, there is no published report of the use of MSL and WLL together in the same case.

Cheng et al reported three cases treated with MSL under local anesthesia (3). They instilled warm saline solution via a syringe in $50-\mathrm{ml}$ aliquots at the orifice of the lobar bronchus and all segments which was removed by suction. They stopped the procedure when the returning fluid became clear or the patient could no longer tolerate the discomfort. They repeated the procedure two to five times, and in each procedure, one lobe was lavaged. The volume of instilled fluid for one lobe was changed from 1700 to $2050 \mathrm{ml}$. In contrast, since our main aim was to prepare patients for WLL, we instilled the same volume of solution, and we discontinued the procedure earlier as the aspirated fluid became bright. After MSL or 'prewash', in both cases, we observed clinical and physiological improvements with an increase in $\mathrm{PaO}_{2}$ level. Thus, WLL was subsequently performed.

There are several important concerns when performing WLL. The first step should include appropriate degassing of the lung to be lavaged. Preoxygenization prior to degassing is very important to ensure replacement of alveolar nitrogen with oxygen, as residual bubbles can diminish the access of lavage fluid to the alveolar space and consequently the efficacy of WLL. In the presented cases, adequate degassing was noted. Second, performing chest percussion can enhance the removal of the accumulated material (18). Hammon et al reported that during WLL, manual chest percussion is superior to mechanical percussion (19). Indeed, in case 1, the initial fluid returns were typically milky, but after the fifth cycle, the fluid became clearer (Figs. 3 and 4). Then, manual chest percussion was performed, and we observed that the manual percussion enhanced the removal of proteinaceous material making the receiving fluids turbid again. Therefore, we strongly recommend manual chest percussion throughout the procedure. We halted the procedure when the returning fluid became clear. After WLL, in both patients, marked clinical and physiological improvements were noted without any serious complications (Fig. 6).

Anesthesia for WLL is a challenging procedure for several reasons. As discussed above, these patients are commonly associated with severe hypoxia increasing the anesthesiaassociated risks. The risk of pneumothorax is also increased. Moreover, single-lung ventilation is required for anesthesia 
for WLL. Single-lung ventilation increases the risk of shunting, hypoxia and carbon dioxide retention. Pre-existing respiratory failure exacerbates the detrimental effects of single-lung ventilation. In addition, alveolar lavage leads to more ventilation-perfusion mismatch. An extensive evaluation is required during the recruiting phase to evaluate whether the patient is able to undergo WLL under general anesthesia. Possible complications should be discussed with the patients and relatives. The anesthesiology team should be familiar with lung separation and single-lung ventilation techniques, possible complications and pathological changes during long-lasting anesthesia procedures (20). In the present cases, patients were strictly examined and evaluated for their ability to undergo general anesthesia. Moreover, after the initiation of general anesthesia, proper monitoring was started and close hemodynamic and respiratory data were obtained. Positioning of the double-lumen tube was confirmed several times during the procedure. These measures were the determining factors for the successful outcome of the patients during the immediate postoperative period. Without any doubt, the successful outcome of WLL requires close collaboration of the thoracic physicians and the anesthesiology team.

In this report, we described our experience using combined lavage techniques for the management of PAP. MSL, or 'prewash', is recommended for hypoxemic patients prior to WLL to improve oxygenation. These two techniques (MSL and WLL) can be used consecutively in patients with PAP who are initially unable to undergo general anesthesia. Several precautions should be taken in the surgical room for the safety and the success of the procedure such as close monitoring and repositioning of the patient, maintenance and inspection of the correct tube position, and manual chest wall percussion.

\section{References}

1. Rosen SH, Castleman B and Liebow AA: Pulmonary alveolar proteinosis. N Engl J Med 258: 1123-1142, 1958.

2. Ramirez J, Nyka W and McLaughlin J: Pulmonary alveolar proteinosis: diagnostic techniques and observations. N Engl J Med 268: 165-171, 1963.

3. Cheng SL, Chang HT, Lau HP, Lee LN and Yang PC: Pulmonary alveolar proteinosis: treatment by bronchofiberscopic lobar lavage. Chest 122: 1480-1485, 2002.

4. Beccaria M, Luisetti M, Rodi G, et al: Long-term durable benefit after whole lung lavage in pulmonary alveolar proteinosis. Eur Respir J 23: 526-531, 2004.
5. Ben-Dov I, Kishinevski Y, Roznman J, et al: Pulmonary alveolar proteinosis in Israel: ethnic clustering. Isr Med Assoc J 1: 75-78, 1999.

6. Ioachimescu OC and Kavuru MS: Pulmonary alveolar proteinosis. Chron Respir Dis 3: 149-159, 2006.

7. Wang BM, Stern EJ, Schmidt RA and Pierson DJ: Diagnosing pulmonary alveolar proteinosis: a review and an update. Chest 111: 460-466, 1997.

8. Davidson JM and Macleod WM: Pulmonary alveolar proteinosis. Br J Dis Chest 463: 13-28, 1969.

9. Larson RK and Gordinier R: Pulmonary alveolar proteinosis: report of six cases, review of the literature and formulation of a new theory. Ann Intern Med 62: 292-312, 1965.

10. Bala RM and Snidal DP: Pulmonary alveolar proteinosis. A case report and review of the literature. Dis Chest 49: 643-651, 1966.

11. Pallav LS, Hansell D, Lawson PR, Reid KB and Morgan C: Pulmonary alveolar proteinosis: clinical aspects and current concepts on pathogenesis. Thorax 55: 67-77, 2000.

12. Kavuru MS, Sullivan EJ, Piccin R, Thomassen MJ and Stoller JK: Exogenous granulocyte-macrophage colony-stimulating factor administration for pulmonary alveolar proteinosis. Am J Respir Crit Care Med 161: 1143-1148, 2000.

13. Seymour JF, Dunn AR, Vincent JM, Persneill JJ and Pain MC: Efficacy of granulocyte-macrophage colony-stimulating factor in acquired alveolar proteinosis. N Engl J Med 335: 1924-1925, 1996.

14. Seymour JF, Persneill JJ, Schoch OD, et al: Therapeutic efficacy of granulocyte-macrophage colony-stimulating factor in patients with idiopathic acquired alveolar proteinosis. Am J Respir Crit Care Med 163: 524-531, 2001.

15. Trapnell BC, Nakata K and Kavuru MS: Pulmonary alveolar proteinosis syndrome. In: Textbook of Respiratory Medicine. 5th edition. Mason RJ, Broaddus VC, Martin TR, King TE, Schraufnagel DE, Murray JF and Nadel JA (eds). Saunders Elsevier, Philadelphia, pp1516-1536, 2010.

16. Prakash UB, Barham SS, Carpenter HA, Dines DE and Marsh HM: Pulmonary alveolar phospholipoproteinosis: experience with 34 cases and a review. Mayo Clin Proc 62: 499-518, 1987.

17. Edis EC, Tabakoglu E, Caglar T, Hatipoglu ON, Cevirme L and Alagol A: Treatment of a primary pulmonary alveolar proteinosis case with severe hypoxaemia by using segmental lavage technique. Ann Acad Med Singapore 36: 871-872, 2007.

18. Perez A and Rogers RM: Enhanced alveolar clearance with chest percussion therapy and positional changes during whole-lung lavage for alveolar proteinosis. Chest 125: 2351-2356, 2004.

19. Hammon WE, McCaffree DR and Cucchiara AJ: A comparison of manual to mechanical chest percussion for clearance of alveolar material in patients with pulmonary alveolar proteinosis (phospholipidosis). Chest 103: 1409-1412, 1993.

20. Webb ST, Evans AJ, Varley AJ and Klein AA: Anaesthesia for serial whole-lung lavage in a patient with severe pulmonary alveolar proteinosis: a case report. J Med Case Reports 2: 360 , 2008. 